\title{
Social Relationship Strength Modulates the Similarity of Brain-to-Brain Representations of Group Members
}

\author{
Taylor D. Guthrie, Youri Y. Benadjaoud, and Robert S. Chavez
}

Department of Psychology, University of Oregon, Eugene, Oregon, USA

\begin{abstract}
Within our societies, humans form cooperative groups with diverse levels of relationship quality among individual group members. In establishing relationships with others, we use attitudes and beliefs about group members and the group as a whole to establish relationships with particular members of our social networks. However, we have yet to understand how brain responses to group members facilitate relationship quality between pairs of individuals. We address this here using a roundrobin interpersonal perception paradigm in which each participant was both a perceiver and target for every other member of their group, in a set of 20 unique groups of between 5 and 6 members in each (total $N=111$ ). Using functional magnetic resonance imaging, we show that measures of social relationship strength modulate the brain-to-brain multivoxel similarity patterns between pairs of participants' responses when perceiving other members of their group in regions of the brain implicated in social cognition. These results provide evidence for a brain mechanism of social cognitive processes serving interpersonal relationship strength among group members.
\end{abstract}

social cognition | MPFC | social neuroscience | MVPA | interpersonal perception

Correspondence: tguthrie@uoregon.edu

NOTE: The current manuscript is a preprint and may not perfectly reflect the final article.

Unlike many other species, humans can be found in cooperative groups for reasons both within and beyond our control. We go to work with colleagues we admire but also with some we dislike. We get adopted into social circles that contain a few close friends but also a handful of less intimate acquaintances. Moreover, our attitudes about the group as a whole can shape our affinity for particular members of that group. For example, two people may bond over their shared enthusiasm for their team or commiserate over their shared frustration with their institutions. Remarkably, groups can remain coherent and productive despite the variable degree of interpersonal closeness found therein. As such, our brains must code for relevant information about our social networks and these processes may reflect or influence our relationships with our fellow group members. Classic work in social psychology has shown that relationships within social networks tend to be largely homophilous, wherein individuals tend to form bonds with others that are similar to themselves on a wide range of demographic and cultural factors (McPherson et al., 2001). This phenomenon has been demonstrated in a wide variety of social network types, from dyadic romantic relationships and friendships to coworker and social groups, and the relationships that form within them (Duncan, 1972; Laumann, 1984; Verbrugge, 1977, 1983). The tendency to interact with others more similar to oneself has the effect of limiting the type of information that one receives and the types of experiences and attitudes that one is introduced to (McPherson et al., 2001). This is thought to contribute to why individuals that have strong social ties tend to share similar political ideologies, values, and beliefs (Lazarsfeld, P. F. \& Merton, R. K., 1954).

More recently, researchers have begun to explore the biological mechanisms that give rise to social connectedness and homophily. For example, previous studies have shown that romantic partners show greater brain-to-brain synchrony in electroencephalogram frequency during social interactions than they do with strangers (Kinreich et al., 2017). Using functional magnetic resonance imaging (fMRI) in a network of peers, other work has shown that individuals with closer social network distances process information more similarly and across larger portions of the cortex in a naturalistic movie watching paradigm (Parkinson et al., 2018).

Together, these lines of work establish a functional role of neural similarity among group members and provide evidence that these mechanisms may contribute to or facilitate interpersonal closeness. However, several questions remain. To understand how similarity in neural responses support interpersonal closeness, it is critical to understand how these systems are engaged when we are thinking about and evaluating real people within our social groups. Indeed, humans have a preference for engaging with socially meaningful content, and contextualizing neural similarity within interpersonal evaluations is important to more closely link these insights with how they operate in the real world (Tamir \& Hughes, 2018). Moreover, attitudes towards groups as a whole can impact the value of interactions with members of that group (Hackel et al., 2017), which may ultimately impact the strength of one's relationship with other group members. Thus, it remains important to understand how the interpersonal similarity in brain responses to group members predicts social relationship strength among pairs of individuals within that group.

In the current study, we used fMRI to test the hypothesis that measures of social relationship strength are related to greater neural similarity in multivoxel response patterns 
when thinking of group members. To do this, we employed a round-robin interpersonal perception paradigm in which each participant was both a perceiver and target for every other member of their group. Both neuroimaging and behavioral measures were collected in preexisting groups of 5-6 people from 20 independent groups of individuals from a variety of real-world social networks. Previous work has established the utility of round-robin fMRI designs in studying social cognition in both univariate (Zerubavel et al., 2015) and multivariate frameworks (Chavez \& Wagner, 2020). Here, we capitalize on the richness of these designs to test whether multiple social relationship metrics-friendship strength, degree of knowing one another, degree of liking one another, and perceived similarity - are related to interpersonal similarity of brain responses when thinking about each of the other individual members of one's group. Furthermore, we examine the same questions when thinking about the group as a whole by capitalizing on the heterogeneity of attitudes towards individual group members to generate aggregated neural responses across group members. Together, these procedures allow us to test how similarity, in both brain representations of individual group members and brain representations of group-level information, between pairs of people are related to the social relationship strength of those individuals.

\section{Methods}

\section{Participants.}

Using a multi-group round-robin design, we recruited a total of 120 right-handed participants between the ages of 18 and 51 (48 females and 72 males) from 20 preexisting independent social network groups including student organizations, local businesses, and friend groups (for a full list of group types see supplementary table 1). Participants ranged in age from 18-51 with a mean age of 23.5 and standard deviation of 7.2. A majority of participants identified as Caucasian (77.9\%), 4.4\% identified as Black or African American, 2.7\% identified as Asian, $1.8 \%$ identified as American Indian or Alaska Native, $1.8 \%$ identified as Native Hawaiian or Pacific Islander, $9.7 \%$ identified as more than one race, $1.8 \%$ chose not to identify their race. A majority of participants identified as non-Hispanic (84.1\%). Six people were recruited from each of the 20 groups and all participants within each group were familiar with one another but had various degrees of closeness with the other members. Across all groups, five participants failed to fulfill scheduled experimental session appointments, two subjects were excluded due to unusable imaging data, and two subjects were excluded for missing or incomplete behavioral data. This left a final $\mathrm{N}=111$ subjects in total with at least five participants per group. All participants were screened for MRI contraindications and had normal or corrected to normal vision. Each participant in the study participated in two sessions. The first was a behavioral ratings session in which participants filled out a series of questionnaires for themselves and their perspective group members. The second session consisted of a scanning session in which participants completed trait judgements for themselves and their peers while undergoing functional neu- roimaging. A schematic of the design used in this study is shown in Figure 1. Participants gave informed consent in both sessions in accordance with the guidelines set by the Internal Review Board at The University of Oregon and were compensated for their participation following each session of the study.

\section{Procedures.}

Behavioral Round-Robin Trait Rating Task. Participants were brought into the lab and asked to answer a series of questions about themselves and a set of known peers from within a small subset of their social network. Each participant was asked to give ratings of interpersonal closeness and similarity to each of the targets. All responses were recorded using PsychoPy stimulus presentation software (Peirce et al., 2019). During the behavioral trait ratings session, participants were presented with an array of scales (responses 1 to 5), each with the name of one of their peers or their own name listed above each. At the top of the screen, a single question was displayed, and participants were asked to give a rating corresponding to the impression of their peers or their evaluation of themselves on that given trait. Participants were required to make a rating for every peer on the screen before being allowed to move on to the next question to ensure complete responses. The entire session lasted approximately one hour. The ratings collected in this session were then used to calculate behavioral interpersonal relationship ratings for use in subsequent fMRI analyses.

The primary metrics used in these analyses were four indices of social relationship strength previously used in a round-robin interpersonal perception paradigm (Chavez \& Wagner, 2020). These indices were based on self-report measures of agreement on a 1-5 Likert scale with a variety of statements about friendship strength ("you are friends with them"), knowing ("you have a good sense of who they are as a person"), liking ("you would like to be closer friends with them"), and perceived similarity ("you think your personality is similar to theirs"). A complete list of the questions used for these metrics can be found in Supplementary Table 2.

Neuroimaging Round-Robin Trait Rating Task. In a separate session, participants were brought back to the lab to complete the fMRI portion of the experiment. While in the scanner, participants were asked to complete a standard traitjudgement task widely used in the study of self and other processing (Kelley et al., 2002; Mitchell et al., 2011). We employed a complete round-robin design, meaning that each participant in the study was both a perceiver and target stimulus for every other participant in the study. Participants were presented with a screen containing two words arranged vertically consisting of white text on a black background. For each trial, the top word displayed either "Self" or the name of one of the other five group members from the same group that the perceiver belonged to. Each perceiver indicated during the behavioral session what name they used most frequently when referring to each of their group members. These were then used in the fMRI portion (e.g., "Jonathan" may have been changed to "John" or the nickname "Jono"). This was 


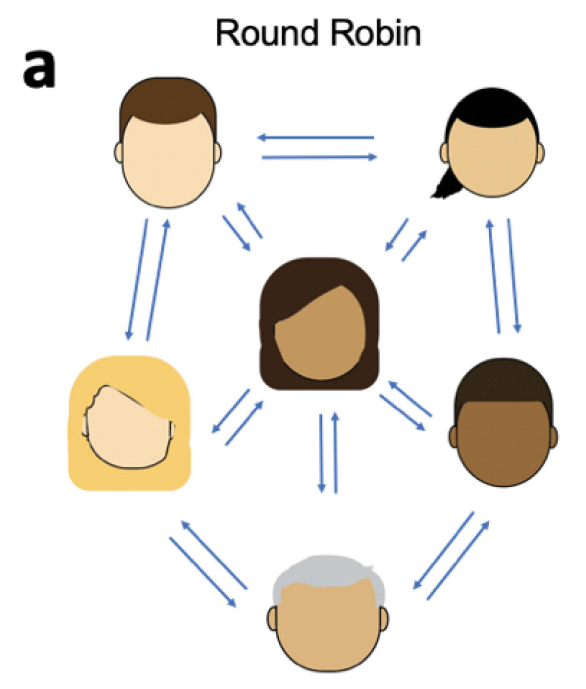

\section{b Parcel Correlations}
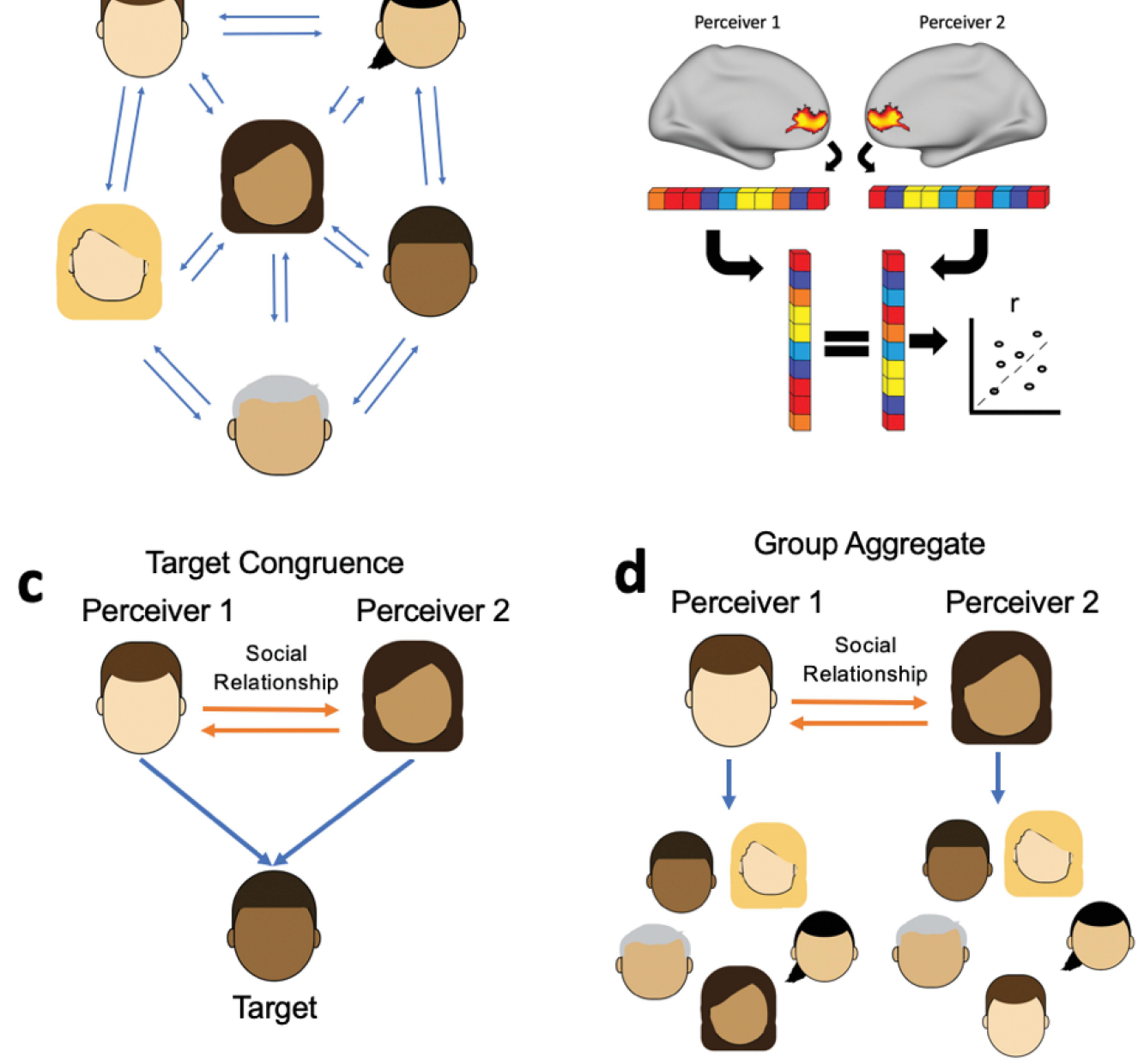

Group Averages

Fig. 1. Schematic of the design employed in the current study. A) We used a round-robin design in which every participant within each group was both a perceiver of and target for every other participant within their group. This design was used to calculate both relationship metrics in the behavioral sessions as well as the neural similarity patterns during brain scanning sessions for each participant in the study. Arrows in the figure indicate direction of social relationship ratings collected during the behavioral session and trait ratings collected during the MRI session. B) For each brain parcel, multivoxel patterns of activity were extracted and these voxel vectors of activity were correlated with one another across subjects using spearman rank correlation. These voxel vectors were representative of either particular members of the perceiver's group or of aggregate representations of the perceiver's group as a whole. C) Brain activity was compared across perceivers for all cases of target congruence (instances where both perceivers were thinking of the same target). The social relationship strength between those perceivers was then used to predict the strength of similarity in multivoxel brain responses in a linear mixed effects model. Each of the social relationship measures were regressed on to target congruent neural correlation values in four separate linear mixed effect models. D) Estimates of each individual group member was averaged for each perceiver and this average representation that each perceiver had of their group was compared with that of the other perceivers of their prospective group. The social relationship strength between those perceivers was again used to predict the strength of similarity in multivoxel brain responses. Each of the social relationship measures were regressed on to group aggregate neural correlation values in four separate linear mixed effect models.

done to ensure that the perceiver did not have to spend additional time in the scanner trying to work out who each target was. The bottom word displayed one of 60 valence-balanced trait adjectives (e.g., "Happy", "Clumsy", "Smart"; Anderson, 1968) for $2000 \mathrm{~ms}$ followed by $2000 \mathrm{~ms}$ of fixation and intermittent passive fixation trials (2000-12000 ms). Jittered trials were optimized using Opseq2 (Dale, 1999). Participants were asked to make either a "yes" or "no" response using a button box in their right hand as to whether or not the trait adjective described either themselves or one of their group members. All targets were presented in each run, and there was a total of twelve trials per target per run in which each target was presented with the same 12 trait-adjectives. The same targets were then used in each subsequent run but were then paired with a new set of 12 trait-adjectives in each one resulting in all targets being paired with all 60 traitadjectives over the course of the experiment. Individual traits were only presented once per target randomly across all runs in the experiment. No two participants were presented with the same target/trait-adjective order across the experiment, to 
account for potential order effects.

Neuroimaging Data Acquisition. Magnetic resonance imaging was conducted with a Siemens 3T Skyra scanner using a 32-channel phased array coil. Structural images were acquired using a T1-weighted MP-RAGE protocol (175 sagittal slices; TR: $2500 \mathrm{~ms}$; TE: $3.43 \mathrm{~ms}$; flip angle: 7; $1 \mathrm{~mm}$ isotropic voxels). Functional images were acquired using a T2*-weighted echo planar sequence (TR: $2000 \mathrm{~ms} ; 72$ axial slices; TE: $25 \mathrm{~ms}$; flip angle: 90; $2 \mathrm{~mm}$ isotropic voxels). For each participant, we collected five runs of the round-robin task (188 whole brain volumes per run). In order to correct for distortion due to B0 inhomogeneity we also acquired a field map (TR: $6390 \mathrm{~ms}$; TE: $47.8 \mathrm{~ms}$; effective echo spacing: $0.345 \mathrm{~ms}$ ). The total length of time for the entire scanning session was approximately 75 minutes and each of the five functional runs were approximately 6 minutes long.

\section{Neuroimaging analysis.}

Round-Robin fMRI Task Preprocessing and Response Pattern Estimation. Functional imaging data for the fMRI task were preprocessed and voxel responses estimated using FSL (Smith et al., 2004). Data were put through a mean-based intensity normalization, high pass filtering (Gaussian-weighted least-squares straight line fitting, with sigma $=100 \mathrm{~s}$ ) and spatially smoothed with a 4mm FWHM Gaussian smoothing kernel. A multi-step normalization procedure was used to register results to standard space. First, functional data were corrected for spatial distortion using a field map unwarping before aligning functional data to each participant's anatomical scan using boundary-based registration (Greve \& Fischl, 2009) in conjunction with a linear registration with FSL's FLIRT tool. These images were then warped to a $2 \mathrm{~mm}$ MNI template using nonlinear registration with FSL's FNIRT tool and a $10 \mathrm{~mm}$ warp field. All first level taskbased analyses were performed in native space before being warped into standard space for final analyses. Parameter estimates were separately estimated for each of the participant's five group members within each of the five runs. These responses were then combined in a second level within-subject fixed-effects analysis yielding parameter estimates for each of the five group members' "target" conditions. Normalized (i.e., z-score) voxel responses for each condition were extracted from a set of 100 parcels (parcellation scheme available: https://neurovault.org/images/39710/), based on metaanalytic coactivation patterns identified from over 10,000 studies in the Neurosynth database (Yarkoni et al., 2011).

Multivariate fMRI Task Target Congruence Analysis. The target congruence analyses investigate social relationship strength between two people when considering one other member of their group at a time (see: Figure 1C). For the round-robin multivoxel pattern analysis, parameter estimates within each region of interest (ROI) were flattened into onedimensional response vectors to allow correlations across participants and conditions. Consistent with previous studies examining the similarity/dissimilarity of neural represen- tations (Kriegeskorte et al., 2008), we computed, for each subject, the dissimilarity between neural response patterns of the peer response vectors from the other participants in the study using Spearman rank correlation distance. Within each parcel, we calculated correlation distances between twoperceivers when making judgements about a third member of their group. This was iterated for all pairwise dyads in the round-robin design and related to social relationship scores obtained in the behavioral session of each perceiver rating the other perceiver of the dyad. We sought to determine whether the target-specific congruence in multivoxel similarity between members of each dyad could be predicted by each of the various social relationship indicators (i.e., "knowing", "friendship", "liking", and "perceived similarity"). In order to account for the nested group structure of the comparisons a series of linear mixed effects models were employed in which the perceiver whose behavioral ratings were being used in each comparison and the group they were nested in was modeled as a random intercept. This procedure was done separately for each of the four relationship strength variables. False discovery rate correction (FDR) was applied to correct for multiple comparisons across the 100 parcel ROIs.

Multivariate fMRI Task Target Congruence Analysis. The target congruence analyses investigate social relationship strength between two people when considering one other member of their group at a time (see: Figure 1C). For the round-robin multivoxel pattern analysis, parameter estimates within each region of interest (ROI) were flattened into onedimensional response vectors to allow correlations across participants and conditions. Consistent with previous studies examining the similarity/dissimilarity of neural representations (Kriegeskorte et al., 2008), we computed, for each subject, the dissimilarity between neural response patterns of the peer response vectors from the other participants in the study using Spearman rank correlation distance. Within each parcel, we calculated correlation distances between twoperceivers when making judgements about a third member of their group. This was iterated for all pairwise dyads in the round-robin design and related to social relationship scores obtained in the behavioral session of each perceiver rating the other perceiver of the dyad. We sought to determine whether the target-specific congruence in multivoxel similarity between members of each dyad could be predicted by each of the various social relationship indicators (i.e., "knowing", "friendship", "liking", and "perceived similarity"). In order to account for the nested group structure of the comparisons a series of linear mixed effects models were employed in which the perceiver whose behavioral ratings were being used in each comparison and the group they were nested in was modeled as a random intercept. This procedure was done separately for each of the four relationship strength variables. False discovery rate correction (FDR) was applied to correct for multiple comparisons across the 100 parcel ROIs.

Multivariate fMRI Task for Group Aggregated Responses.. People sometimes think differently about groups collectively than they do about the individual members within them. As 
such, we were also interested in investigating how average representations of each participant's group as a whole were predicted by social relationship strength between pairs of participants (see: Figure 1D). Mirroring the process described above in the target congruence analysis, for each perceiver multivoxel parameter estimates within each ROI were flattened into one-dimensional response vectors for each target individually. Then, response vectors were averaged at each voxel within the vector and across all other targets in each perceiver's group to create a single aggregated onedimensional vector representing the group aggregated response. It is important to note that this analysis is fundamentally different than the original target congruence analysis in that each perceiver's aggregate group representation includes the partner of the dyad from each comparison that is being made. This was to ensure that the aggregate was more representative of the group as a whole and not merely an average of the individual instances that were already compared in the original target congruence analysis. Spearman rank correlation distances of brain responses were then calculated in each parcel using the group averaged vectors for each set of perceivers within a group. As in the target congruence analyses, the interpersonal closeness ratings that perceiver one selected in regard to perceiver two were aligned with each corresponding neural dissimilarity value. The same four linear mixed effects models described earlier for the social relationship variables were then applied here as well. False discovery rate corrections were made to correct for multiple comparisons across the 100 parcels.

Supplementary Analyses. Due to moderate correlation strength between the social relationship variables that are used in the individual linear mixed effects models, additional analyses were run in which the four relationship categories were collapsed into one variable representing overall relationship strength. This aggregate social relationship rating was then regressed on the neural congruence value in the same way that the individual relationship metrics were in the above analyses. Separate linear mixed effects models were constructed for the target congruence and group aggregate neural congruence values as was done with the individual social relationship measures. The results from these analyses are highly consistent with the results obtained from modeling the separate relationship metrics individually and can be found in Supplementary Figures 1 and 2.

It was also pertinent to test whether similarities in trait responses while in the MRI were related in some way to the target congruence neural similarity metrics used in the aforementioned modeling procedures. Response values for all trait-adjective words that perceiver one and perceiver two indicated for a particular congruent target were extracted. All rows with missing responses were excluded and a correlation was calculated to determine how similar the responses of the two perceivers were for that same target. This response correlation value was then regressed on the neural congruence correlation value that was obtained from the two perceivers both thinking of the same target that the responses were acquired for. Results for this analysis can be found in Supplementary
Figure 3.

\section{Results}

\section{Social Relationship Strength and Neural Similarity to In-} dividual Group Members.

A schematic of the design used in this study is shown in Figure 1. All analyses were performed in the same manner within each of the 100 parcels independently and significance values were corrected for multiple comparisons using FDR. All fMRI results are reported with FDR-corrected p-values. Significant regions from these analyses are displayed in Figure 2, and the significant clusters within brain regions commonly associated with person knowledge and social cognition are described in detail below. Some regions exhibited an inverse relationship such that higher levels of social relationship strength were associated with lower levels of brain activity similarity. These regions were largely found in parts of the brain associated with sensory or motor processes but were inconsistent across the relationship metrics. A complete list of all significant cluster statistics for the individual target results can be found in Supplementary Table 3. Additionally, correlation strength between each of the various social relationship variables that are used in the linear mixed effects models can be found in Supplementary Table 5.

Friendship. Results from the linear mixed effect model showed that friendship was a significant predictor of multivoxel brain similarity when both perceivers were thinking of the same target. The original hypothesis posited that higher levels of friendship would result in brain responses to specific targets between perceivers that were more similar, specifically within regions known to be involved in social cognition and person perception. Consistent with this hypothesis, it was found that higher levels of friendship between perceivers was significantly associated with higher levels of multivoxel similarity within the dMPFC after all significance values had been corrected for multiple comparisons with a false discovery rate method $(\mathrm{b}=-.021, \mathrm{SE}=.017, \mathrm{p}<.001)$. Similarly, the anterior insula also showed the pattern that higher levels of friendship indicated higher levels of similarity between distributed brain responses $(b=-.017, \mathrm{SE}=.019, \mathrm{p}<.001)$.

Knowing. As was done above for friendship scores, ratings about how much each perceiver knew the other perceiver in each dyad were used as predictors of target congruence brain response similarity. It was predicted that social brain regions would show higher degrees of multivoxel similarity for pairs of perceivers that indicated that they knew each other better than ones that indicated otherwise. Consistent with this hypothesis, higher degrees of multivoxel similarity in the $\mathrm{dMPFC}(\mathrm{b}=-.005, \mathrm{SE}=.018, \mathrm{p}<.001)$ and the anterior insula $(\mathrm{b}=-.003, \mathrm{SE}=.020, \mathrm{p}=.030)$ were associated with higher levels of knowing after corrections for multiple comparisons. Given its role in self-referential processing (Wagner et al., 2012), it is notable that we found an inverse relationship in the vMPFC, such that higher degrees of knowing 


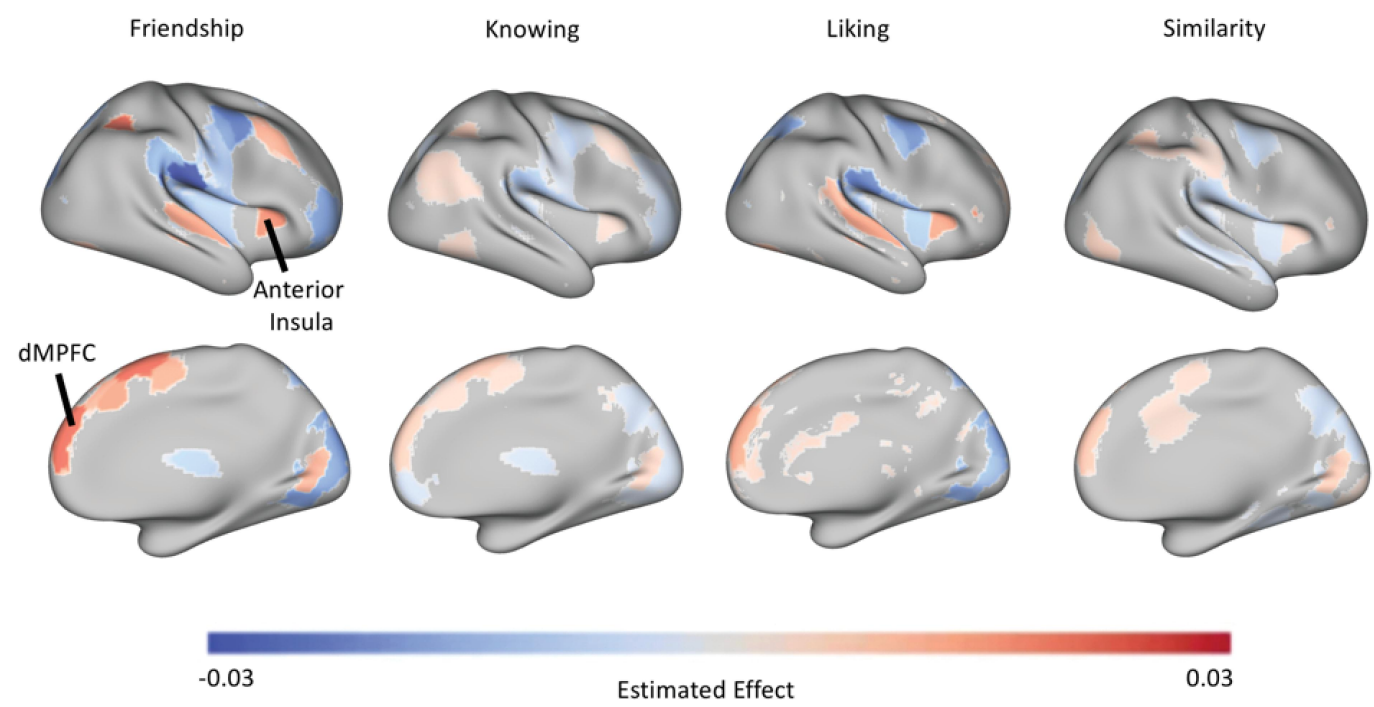

Fig. 2. Brain regions in which there was a significant relationship between social relationship metrics and similarity in brain response patterns when thinking of other group members. Warm colors indicate positive associations such that greater neural similarity to the group members was related to stronger relationship metrices; cool colors indicated where there were negative associations. Positive associations across relationship metrics were consistently found throughout brain regions commonly associated with person perception and social cognition, including the dorsal medial prefrontal cortex and the anterior insula.

between individuals was associated with lower levels of multivoxel similarity $(\mathrm{b}=.003, \mathrm{SE}=.028, \mathrm{p}=.042)$.

Liking. The original hypothesis also posited that higher degrees of liking between perceivers would result in higher degrees of similarity between voxel responses within social brain regions when perceivers were tasked to think of the same target. Consistent with this hypothesis, it was found that higher scores of liking between perceivers resulted in higher levels of similarity of multivoxel activity patterns within the $\mathrm{dMPFC}(\mathrm{b}=-.013, \mathrm{SE}=.017, \mathrm{p}=.002)$ and the anterior insula $(b=-.014, \mathrm{SE}=.020, \mathrm{p}=.010)$.

Perceived Similarity. The degree to which each perceiver thought that they were similar to the other of each dyad was also used as a predictor of target congruence brain response similarity. It was hypothesized that higher degrees of perceived similarity would result in higher levels of multivoxel similarity in social brain regions. Consistent with this hypothesis, we found that higher levels of multivoxel similarity in the dMPFC $(b=-.008, \mathrm{SE}=.016, \mathrm{p}<.001)$ and anterior insula $(b=-.005, S E=.017, p=.017)$ were associated with higher levels of perceived similarity between individuals in each dyad after corrections for multiple comparisons were made.

\section{Social Relationship Strength and Neural Similarity to the Group Aggregate.}

Next, we investigated if social relationship strength between participants was related to similarity in brain representations when considering the group as a whole. Rather than calculating similarity between brain responses to congruent targets, here these responses were calculated within each perceiver by aggregating multivoxel responses when they were making trait-judgments of about every member of their group. These average multivoxel activity patterns were used to reflect group-level representations within each perceiver and were then compared in a dyadic fashion across every member of each group. All other analytic procedures mirror the individual target analyses, including the linear effects modeling, use of parcellation schemes, and multiple comparisons using FDR. A complete list of all significant cluster statistics for the group-aggregated results can be found in Supplementary Table 4.

Friendship. Using friendship strength as a predictor for averaged group multivoxel similarity, it was found that a significant relationship again existed in the dMPFC $(b=-.021$, $\mathrm{SE}=.026, \mathrm{p}=.026)$ with higher levels of friendship indicating higher levels of multivoxel similarity within the region. This was similar to what was seen above in this same region for target congruent multivoxel similarity and friendship strength. One other region in somatosensory cortex not known to be involved in social cognition or person perception showed an inverse relationship such that higher friendship ratings indicated lower levels of similarity between brain responses $(b=0.025, \mathrm{SE}=0.007, \mathrm{p}=0.048)$.

Knowing. It was hypothesized that there would be higher levels of multivoxel similarity for averaged group activity in social brain regions between perceivers that indicated they knew each other more strongly. Consistent with this hypothesis, higher ratings of knowing were significantly associated with higher levels of multivoxel similarity within the dMPFC $(\mathrm{b}=-.006, \mathrm{SE}=.028, \mathrm{p}=.011)$ after correcting for multiple comparisons 


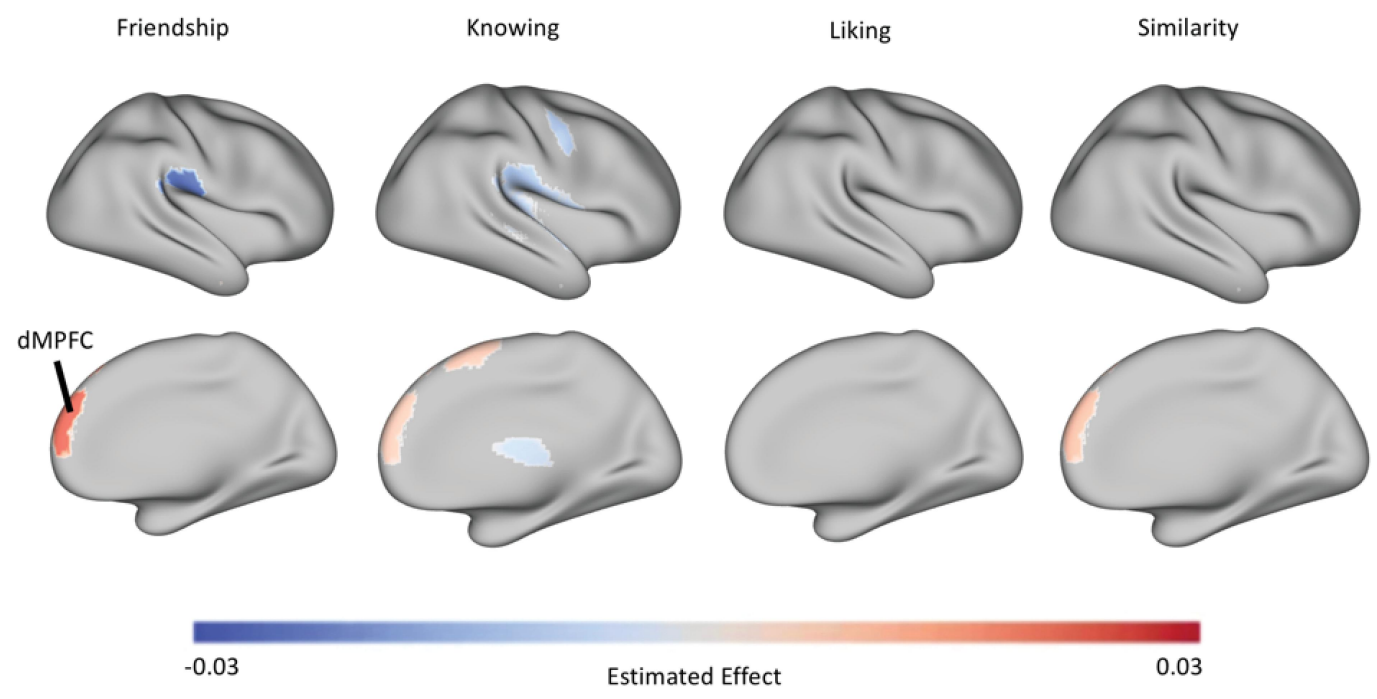

Fig. 3. Results when relating social relationship metrics between pairs of subjects to brain similarity patterns to a group representation calculated by aggregating across all other members of the group. Warm colors indicate a positive relationship such that greater neural similarity to the group was related to stronger relationship metrices, and cool colors where there was negative relationship. For these analyses, only the dorsal medial prefrontal cortex was found across multiple relationship metrics, and there were no significant results for the metric liking.

Liking. After multiple comparison corrections, no regions showed a significant relationship between levels of liking between perceivers and group averaged multivoxel similarity in the brain responses. This was the only metric across all tested that did not show a significant relationship in brain similarity patterns between perceivers in the dMPFC. Moreover, this was the only metric across all tested that did not show a relationship to brain similarity patterns in any region of the brain after correcting for multiple comparisons.

Perceived Similarity. Lastly, it was hypothesized that higher degrees of perceived similarity would be associated with higher levels of group averaged multivoxel similarity within social brain regions. This was found to be true in the dMPFC $(\mathrm{b}=-.009, \mathrm{SE}=.023, \mathrm{p}=.001)$ such that higher levels of perceived similarity were significantly associated with higher levels of group averaged brain responses. This was the only region that survived false discovery rate correction.

\section{Discussion}

The attitudes and beliefs we have about others in our social networks influence not only how we behave but also the quality of our relationships. Here, we show that multiple measures of relationship strength between pairs of individuals are related to multivoxel similarity patterns of brain responses when those people are considering the traits of other members of their group. Across all measures of relationship quality (i.e., friendship strength, degree of knowing, degree of liking, and perceived similarity), the most consistently implicated brain areas included the dMPFC and anterior insula where greater similarity in brain responses to individual group members was related to greater relationship quality. Furthermore, when considering the group as a whole by aggregating across brain responses to individual members, we show that the same relationship quality metrics, except for the degree of liking, were also related to multivoxel dMPFC similarity between pairs of participants.

Relative to other brain areas that are also involved in social cognition, such as the temporal parietal junction and posterior cingulate cortex, the $\mathrm{dMPFC}$ has been shown to be more involved in the formation of impressions of others and in trait attribution (Mitchell, 2004). Studies have shown that the dMPFC is involved when considering the traits of others (Todorov et al., 2007) as well as in making inferences about others when only given small amounts of information (Jenkins \& Mitchell, 2010; Mitchell, 2009). Other work has similarly demonstrated that familiar vs. unfamiliar faces could be decoded within the $\mathrm{dMPFC}$ and that the decoding model could be applied to new sets of familiar and unfamiliar faces that were not used to train the model (Visconti di Oleggio Castello et al., 2017). Recently, researchers have demonstrated that this region also encodes information about others' beliefs using single unit recordings in human patients (Jamali et al., 2021). Indeed, meta-analyses have supported the role of the involvement of the dMPFC in representing person knowledge-including familiarity, similarity, and intimacy with the social target in multiple stimulus paradigms - more generally (Denny et al., 2012; Wagner et al., 2012). Our results provide additional evidence that the dMPFC is involved in person knowledge but extend these findings to demonstrate that these response patterns are shared across people when considering fellow group members both individually and as a group. In turn, both of these patterns are consistently related to relationship strength across all metrics measured. Other brain regions involved in social cognition did not show the same consistency across relationship strength metrics, pointing to a central role of the dMPFC in supporting these processes.

Beyond the dMPFC, the current study showed that the 
anterior insula was also consistently related to relationship strength when considering other group members individually. Using a computational model of trail-by-trial measures of learned dyadic similarity between participants and social targets, previous work has shown that activation in the anterior insula predicted participants' choices in who they would choose as an 'ally' when making judgments about agreement on a politically charged issue (Lau et al., 2020). These results support the idea that this region supports social-structure learning and is based on general principles of structure learning that are even present in early development (Gershman \& Cikara, 2020). Our results dovetail with these previous findings, by showing that the strength of real-world relationships is reflected in shared neural representation of other individuals within our close-knit social groups. However, relationship strength was not shown to be related to pattern similarity in the anterior insula when considering the group as a whole. This discrepancy may reflect differences in thinking about individuals versus groups of people, but also may be due to multiple differences in methodology between the current study and previous investigations, including the use of real-world targets in the current study and trial-by-trial estimates in the previous ones. Nonetheless, our results suggest that interpersonal relationship strength between two people is related to the degree to which there is similarity in anterior insula responses when thinking of their fellow group members individually.

Some regions of the brain in the current study showed an inverse relationship with regards to social relationship strength and neural similarity patterns. These regions were largely found in sensory and motor regions and tended to be inconsistent across the relationship metrics. Though difficult to interpret in the current context, it is possible that these patterns may reflect broad differences in the roles of these different regions and may suggest a need to separate similar related stimuli in the real world into distinguishable patterns for different processing purposes. The idea that distinct neural circuitry may create distinguishable patterns of activity for objects or scenes that are similar was initially proposed by David Marr (Marr, 1969) and James Albus (Albus, 1971) as a strategy for increasing the signal to noise ratio to in turn be better at distinguishing subtle differences in the environment. This phenomenon has been demonstrated in hippocampal and dentate gyrus regions and in areas of the cerebral cortex (Cayco-Gajic \& Silver, 2019; Yassa \& Stark, 2011). Although it is not evident whether other broad regions or networks are capable of achieving the same outcome, it could serve as another route by which the brain differentiates close or similar others.

A strength of the current study is the use of the roundrobin design to capture real-world social relationship processing in brain-to-brain similarity patterns between closeknit individuals. Typical neuroimaging studies of person knowledge either use multiple social target stimuli that are highly recognizable and consistent across participants, such as political figures (Kelley et al., 2002) and celebrities (Thornton \& Mitchell, 2017), but interpersonally unknown to the participants themselves. Other studies use more intimate relationships, such as a participant's best friend or family member (Chavez et al., 2017), but targets remain idiosyncratic across participants. The round-robin design employed here captures the strengths of both approaches by allowing for well-known targets that are consistent across all members of a group. Furthermore, having multiple targets within each group allowed us to test brain-to-brain multivoxel similarity patterns among all pairwise members of each group and relate them to social relationship metrics in a way not possible in other designs with fictional characters or single targets.

Other studies have surveyed complete social networks for behavioral measures and subsampling for neuroimaging measures (Parkinson et al., 2017, 2018). These studies aimed to broadly characterize relationships in larger networks, but due to logistical limitations may not be able to capture more detailed information about individual relationships among individuals within those networks. For example, a previous study showed that social network distance was related to time-series similarity in activation among different brain regions when participants viewed video clips that varied widely in content to broadly capture the possible stimulus space (Parkinson et al., 2018). The results of the current study are consistent with these previous findings and extends these ideas to show that similar effects of homophily effects are reflected in multivoxel brain activity patterns when subjects are directly considering the traits of other individuals in groups and using multiple relationship strength metrics in a variety of kinds of social groups.

In conclusion, our results show that multiple measures of social relationship strength between individuals are related to their shared brain pattern similarity when thinking of the behaviors and traits of their fellow group members. These results were most consistently shown within regions of the brain associated with social cognition. Furthermore, we found similar results when estimating subjects' representations of their group as a whole when aggregating across brain responses to individual members of that group. Together, these results suggest that that social relationship strength with members of our social networks may be served by brain mechanisms that facilitate similar responses patterns when thinking of fellow group members and our group as a whole.

\section{ACKNOWLEDGEMENTS}

Research funded by University of Oregon. The authors would like to acknowledge Moriah Stendel, Faith Collins and Angela O'Neil for helping with data collection.

\section{OPEN SCIENCE PRACTICES}

Data, materials, and code used to generate the findings in this study are openly available via an Open Science Framework repository at https://osf.io/jwxph/.

\section{References}

Albus, J. S. (1971). A theory of cerebellar function. Mathematical Biosciences, 10(1-2), 25-61. https://doi.org/10.1016/0025-5564(71)90051-4

Anderson, N. H. (1968). Likableness ratings of 555 personality-trait words. Journal of Personality and Social Psychology, 9(3), 272-279.

Cayco-Gajic, N. A., \& Silver, R. A. (2019). Re- 
evaluating Circuit Mechanisms Underlying Pattern Separation. Neuron, 101(4), 584-602. https://doi.org/10.1016/j.neuron.2019.01.044

Chang, L. J., Jolly, E., Cheong, J. H., Rapuano, K. M., Greenstein, N., Chen, P.-H. A., \& Manning, J. R. (2020). Endogenous variation in ventromedial prefrontal cortex state dynamics during naturalistic viewing reflects affective experience. BioRxiv, 487892. https://doi.org/10.1101/487892

Chavez, R. S., Heatherton, T. F., \& Wagner, D. D. (2017). Neural Population Decoding Reveals the Intrinsic Positivity of the Self. Cerebral Cortex, 27(11), 5222-5229. https://doi.org/10.1093/cercor/bhw302

Chavez, R. S., \& Wagner, D. D. (2020). The neural representation of self is recapitulated in the brains of friends: A round-robin fMRI study. Journal of Personality and Social Psychology, 118(3), 407-416. https://doi.org/10.1037/pspa0000178

Dale, A. M. (1999). Optimal experimental design for event-related fMRI. Human Brain Mapping, 8(2-3), 109-114. https://doi.org/10.1002/(SICI)10970193(1999)8:2/3<109::AID-HBM7>3.0.CO;2-W

Denny, B. T., Kober, H., Wager, T. D., \& Ochsner, K. N. (2012). A meta-analysis of functional neuroimaging studies of self- and other judgments reveals a spatial gradient for mentalizing in medial prefrontal cortex. Journal of Cognitive Neuroscience, 24(8), 1742-1752. https://doi.org/10.1162/jocn ${ }_{a 0} 0233$

Duncan, R. B. (1972). Characteristics of Organizational Environments and Perceived Environmental Uncertainty. Administrative Science Quarterly, 17(3), 313. https://doi.org/10.2307/2392145

Gershman, S. J., \& Cikara, M. (2020). SocialStructure Learning. Current Directions in Psychological Science, 29(5), 460-466. https://doi.org/10.1177/0963721420924481

Greve, D. N., \& Fischl, B. (2009). Accurate and robust brain image alignment using boundarybased registration. NeuroImage, 48(1), 63-72. https://doi.org/10.1016/j.neuroimage.2009.06.060

Hackel, L. M., Zaki, J., \& Van Bavel, J. J. (2017). Social identity shapes social valuation: Evidence from prosocial behavior and vicarious reward. Social Cognitive and Affective Neuroscience, 12(8), 1219-1228. https://doi.org/10.1093/scan/nsx045

Jamali, M., Grannan, B. L., Fedorenko, E., Saxe, R., BáezMendoza, R., \& Williams, Z. M. (2021). Singleneuronal predictions of others' beliefs in humans. Nature, 1-5. https://doi.org/10.1038/s41586-021-03184-0

Jenkins, A. C., \& Mitchell, J. P. (2010). Mentalizing under uncertainty: Dissociated neural responses to ambiguous and unambiguous mental state inferences. Cerebral Cortex, 20(2), 404-410.

Kelley, W. M., Macrae, C. N., Wyland, C. L., Caglar, S., Inati, S., \& Heatherton, T. F. (2002). Finding the self? An event-related fMRI study. Journal of Cognitive Neuroscience, 14(5), 785-794.
Kinreich, S., Djalovski, A., Kraus, L., Louzoun, Y., \& Feldman, R. (2017). Brain-to-Brain Synchrony during Naturalistic Social Interactions. Scientific Reports, 7(1), 17060. https://doi.org/10.1038/s41598-017-17339-5

Kriegeskorte, N., Mur, M., \& Bandettini, P. A. (2008). Representational similarity analysis-Connecting the branches of systems neuroscience. Frontiers in Systems Neuroscience, 2. https://doi.org/10.3389/neuro.06.004.2008

Lau, T., Gershman, S. J., \& Cikara, M. (2020). Social structure learning in human anterior insula. ELife, 9, e53162. https://doi.org/10.7554/eLife.53162

Laumann, E. O. (1984). Detroit Area Study, 1966: Stratified Association and Values in the Urban Community: Version 3 [Data set]. ICPSR - Interuniversity Consortium for Political and Social Research. https://doi.org/10.3886/ICPSR07405.V3

Lazarsfeld, P. F., \& Merton, R. K. (1954). Friendship as a social process: A substantive and methodological analysis. Freedom and Control in Modern Society, 18(1), 18-66.

Marr, D. (1969). A theory of cerebellar cortex. The Journal of Physiology, 202(2), 437-470. https://doi.org/10.1113/jphysiol.1969.sp008820

McPherson, M., Smith-Lovin, L., \& Cook, J. M. (2001). Birds of a Feather: Homophily in Social Networks. Annual Review of Sociology, 27(1), 415-444. https://doi.org/10.1146/annurev.soc.27.1.415

Mitchell, J. P. (2004). Encoding-Specific Effects of Social Cognition on the Neural Correlates of Subsequent Memory. Journal of Neuroscience, 24(21), 4912-4917. https://doi.org/10.1523/JNEUROSCI.0481-04.2004

Mitchell, Jason P. (2009). Inferences about mental states. Philosophical Transactions of the Royal Society of London. Series B, Biological Sciences, 364(1521), 1309-1316. https://doi.org/10.1098/rstb.2008.0318

Mitchell, Jason P, Schirmer, J., Ames, D. L., \& Gilbert, D. T. (2011). Medial prefrontal cortex predicts intertemporal choice. Journal of Cognitive Neuroscience, 23(4), 857-866. https://doi.org/10.1162/jocn.2010.21479

Parkinson, C., Kleinbaum, A. M., \& Wheatley, T. (2017). Spontaneous neural encoding of social network position. Nature Human Behaviour, 1(5), 0072. https://doi.org/10.1038/s41562-017-0072

Parkinson, C., Kleinbaum, A. M., \& Wheatley, T. (2018). Similar neural responses predict friendship. Nature Communications, 9(1), 332. https://doi.org/10.1038/s41467-017-02722-7

Peirce, J., Gray, J. R., Simpson, S., MacAskill, M., Höchenberger, R., Sogo, H., Kastman, E., \& Lindeløv, J. K. (2019). PsychoPy2: Experiments in behavior made easy. Behavior Research Methods, 51(1), 195-203. https://doi.org/10.3758/s13428-018-01193-y

Smith, S. M., Jenkinson, M., Woolrich, M. W., Beckmann, C. F., Behrens, T. E. J., Johansen-Berg, H., Bannister, P. R., De Luca, M., Drobnjak, I., Flitney, D. E., Niazy, R. K., Saunders, J., Vickers, J., Zhang, Y., De Stefano, 
N., Brady, J. M., \& Matthews, P. M. (2004). Advances in functional and structural MR image analysis and implementation as FSL. NeuroImage, 23, S208-S219. https://doi.org/10.1016/j.neuroimage.2004.07.051

Tamir, D. I., \& Hughes, B. L. (2018). Social Rewards: From Basic Social Building Blocks to Complex Social Behavior. Perspectives on Psychological Science, 13(6), 700-717. https://doi.org/10.1177/1745691618776263

Familiar People. Journal of Cognitive Neuroscience, 29(9), 1583-1594. https://doi.org/10.1162/jocn ${ }_{a 0} 1151$

Todorov, A., Gobbini, M. I., Evans, K. K., \& Haxby, J. V. (2007). Spontaneous retrieval of affective person knowledge in face perception. Neuropsychologia, 45(1), 163-173. https://doi.org/10.1016/j.neuropsychologia.2006.04.018

Verbrugge, L. M. (1977). The Structure of Adult Friendship Choices. Social Forces, 56(2), 576. https://doi.org/10.2307/2577741

Verbrugge, L. M. (1983). Multiple Roles and Physical Health of Women and Men. Journal of Health and Social Behavior, 24(1), 16. https://doi.org/10.2307/2136300

Visconti di Oleggio Castello, M., Halchenko, Y. O., Guntupalli, J. S., Gors, J. D., \& Gobbini, M. I. (2017). The neural representation of personally familiar and unfamiliar faces in the distributed system for face perception. Scientific Reports, 7(1), 12237. https://doi.org/10.1038/s41598-017-12559-1

Wagner, D. D., Haxby, J. V., \& Heatherton, T. F. (2012). The Representation of Self and Person Knowledge in the Medial Prefrontal Cortex. Wiley Interdisciplinary Reviews: Cognitive Science, 3(4), 451-470. https://doi.org/10.1002/wcs.1183

Yarkoni, T., Poldrack, R. A., Nichols, T. E., Essen, D. C. V., Wager, T. D., \& Van Essen, D. C. (2011). Large-scale automated synthesis of human functional neuroimaging data. Nature Methods, 8(8), 665-670. https://doi.org/10.1038/NMETH.1635

Yassa, M. A., \& Stark, C. E. L. (2011). Pattern separation in the hippocampus. Trends in Neurosciences, 34(10), 515-525. https://doi.org/10.1016/j.tins.2011.06.006

Zerubavel, N., Bearman, P. S., Weber, J., \& Ochsner, K. N. (2015). Neural mechanisms tracking popularity in real-world social networks. Proceedings of the National Academy of Sciences, 112(49), 15072-15077. https://doi.org/10.1073/pnas.1511477112 


\section{SUPPLEMENTARY MATERIALS}

Social relationship strength modulates the similarity of brain-to-brain representations of group members

*Taylor D. Guthrie, Youri Y. Benadjaoud, \& Robert S. Chavez

Department of Psychology, University of Oregon, Eugene, OR, USA

Supplementary Table 1

Groups by Type of Social Network

\begin{tabular}{lcc} 
Type of Social Network & N (groups) & Percentage (\%) \\
\hline Academic Organizations & 5 & 25 \\
Social Organizations & 7 & 35 \\
Coworkers & 4 & 20 \\
Friends & 4 & 20 \\
Total & 20 & 100
\end{tabular}

Supplementary Table 2

Survey Items for Social Relationship Strength Measures

Social Relationship Variable Survey Item

Friendship

You are friends with them

Knowing

You feel like you know them 
You feel like you know how they think

You have a good sense of who they are as a person

You would be able to predict how they would act in a situation

Liking

You would like to be closer friends with them

Perceived Similarity

You Identify with them

You are similar to them

You think your personality is similar to theirs

Supplementary Table 3

Target Congruence Linear Mixed Effects Model Output for Significant Parcels

\begin{tabular}{llllll}
\hline & & & & \\
Social Relationship Variable & Parcel Number & Brain Region & $b$ & $S E$ & $p$ \\
\hline Friendship & parc1 & premotor cortex & 0.022 & 0.005 & $<.001$ \\
& parc6 & visual cortex & 0.017 & 0.003 & $<.001$ \\
& parc10 & fusiform & -0.009 & 0.003 & 0.029 \\
& parc14 & supplementary motor cortex & -0.021 & 0.004 & $<.001$ \\
& parc15 & posterior insula & 0.008 & 0.003 & 0.034 \\
& parc22 & superior temporal cortex & -0.013 & 0.003 & 0.001 \\
& parc24 & intraparietal sulcus & 0.011 & 0.004 & 0.028 \\
& parc26 & dorsal medial prefrontal cortex & -0.021 & 0.003 & $<.001$ \\
& parc33 & somatosensory cortex & 0.029 & 0.004 & $<.001$ \\
& parc34 & temporal parietal junction & 0.014 & 0.005 & 0.011 \\
& parc37 & lingual gyrus & -0.011 & 0.004 & 0.028 \\
& parc40 & supplementary motor area & -0.014 & 0.004 & 0.008 \\
& parc41 & premotor cortex & -0.012 & 0.004 & 0.020 \\
& parc44 & lateral inferior frontal cortex & -0.012 & 0.004 & 0.014 \\
& parc51 & anterior insula & -0.017 & 0.004 & $<.001$ \\
& parc57 & auditory cortex & 0.011 & 0.003 & 0.003 \\
parc65 & early visual cortex & 0.016 & 0.003 & $<.001$ \\
parc69 & dorsal lateral prefrontal cortex & 0.015 & 0.004 & 0.001 \\
& parc73 & primary motor cortex & 0.009 & 0.003 & 0.029 \\
parc81 & somatosensory cortex & 0.012 & 0.004 & 0.004 \\
parc90 & premotor cortex & 0.017 & 0.004 & 0.001 \\
parc91 & intra parietal lobule & -0.022 & 0.005 & $<.001$
\end{tabular}


Knowing

$\begin{array}{llccc}\text { parc1 } & \text { premotor cortex } & 0.004 & 0.001 & 0.037 \\ \text { parc6 } & \text { visual cortex } & 0.003 & 0.001 & 0.019 \\ \text { parc14 } & \text { supplementary motor cortex } & -0.007 & 0.001 & <.001 \\ \text { parc23 } & \text { ventral medial prefrontal cortex } & 0.003 & 0.001 & 0.042 \\ \text { parc25 } & \text { primary motor cortex } & -0.003 & 0.001 & 0.006 \\ \text { parc26 } & \text { dorsal medial prefrontal cortex } & -0.006 & 0.001 & <.001 \\ \text { parc30 } & \text { precuneus } & 0.003 & 0.001 & 0.020 \\ \text { parc32 } & \text { occiptotemporal cortex } & -0.004 & 0.001 & 0.014 \\ \text { parc33 } & \text { somatosensory cortex } & 0.009 & 0.001 & <.001 \\ \text { parc37 } & \text { lingual gyrus } & -0.005 & 0.001 & 0.001 \\ \text { parc40 } & \text { supplementary motor area } & -0.004 & 0.001 & 0.019 \\ \text { parc41 } & \text { premotor cortex } & -0.004 & 0.001 & 0.014 \\ \text { parc44 } & \text { lateral inferior frontal cortex } & -0.003 & 0.001 & 0.014 \\ \text { parc51 } & \text { anterior insula } & -0.003 & 0.001 & 0.030 \\ \text { parc56 } & \text { dorsal lateral prefrontal cortex } & 0.003 & 0.001 & 0.020 \\ \text { parc57 } & \text { auditory cortex } & 0.005 & 0.001 & <.001 \\ \text { parc64 } & \text { intraparietal sulcus } & 0.004 & 0.001 & 0.020 \\ \text { parc65 } & \text { early visual cortex } & 0.003 & 0.001 & 0.014 \\ \text { parc69 } & \text { dorsal lateral prefrontal cortex } & 0.004 & 0.001 & 0.000 \\ \text { parc73 } & \text { primary motor cortex } & 0.003 & 0.001 & 0.024 \\ \text { parc76 } & \text { inferior occipital cortex } & 0.003 & 0.001 & 0.048 \\ \text { parc78 } & \text { temporal parietal junction } & -0.004 & 0.001 & 0.030 \\ \text { parc81 } & \text { somatosensory cortex } & 0.006 & 0.001 & <.001 \\ \text { parc89 } & \text { temporal parietal junction } & -0.004 & 0.001 & 0.014 \\ \text { parc90 } & \text { premotor cortex } & 0.006 & 0.001 & <.001 \\ \text { parc91 } & \text { intra parietal lobule } & -0.006 & 0.001 & <.001 \\ \text { parc92 } & \text { thalamus } & 0.004 & 0.001 & <.001\end{array}$

Liking

parc1
parc6
parc9
parc10
parc18
parc22
parc24

premotor cortex

visual cortex

inferior frontal cortex

fusiform

intra parietal sulcus

superior temporal cortex

intra parietal sulcus $\begin{array}{lll}0.017 & 0.005 & 0.005\end{array}$

$\begin{array}{llll}0.015 & 0.003 & <.001\end{array}$

$\begin{array}{lll}-0.016 & 0.005 & 0.015\end{array}$

$\begin{array}{lll}-0.011 & 0.003 & 0.013\end{array}$

$\begin{array}{lll}0.015 & 0.005 & 0.037\end{array}$

$\begin{array}{lll}-0.013 & 0.003 & 0.002\end{array}$

$\begin{array}{lll}0.012 & 0.004 & 0.019\end{array}$ 


$\begin{array}{llccc}\text { parc25 } & \text { primary motor cortex } & 0.012 & 0.004 & 0.008 \\ \text { parc26 } & \text { dorsal medial prefrontal cortex } & -0.013 & 0.003 & 0.002 \\ \text { parc27 } & \text { planum temporale } & -0.010 & 0.003 & 0.025 \\ \text { parc33 } & \text { somatosensory cortex } & 0.022 & 0.004 & <.001 \\ \text { parc51 } & \text { anterior insula } & -0.014 & 0.004 & 0.010 \\ \text { parc57 } & \text { auditory cortex } & 0.010 & 0.003 & 0.013 \\ \text { parc65 } & \text { early visual cortex } & 0.012 & 0.003 & 0.006 \\ \text { parc71 } & \text { insular cortex } & 0.009 & 0.003 & 0.047 \\ \text { parc81 } & \text { somatosensory cortex } & 0.013 & 0.004 & 0.003 \\ \text { parc90 } & \text { premotor cortex } & 0.012 & 0.004 & 0.025 \\ \text { parc95 } & \text { anterior cingulate cortex } & -0.005 & 0.002 & 0.047\end{array}$

Perceived Similarity

$\begin{array}{llccc}\text { parc1 } & \text { premotor cortex } & 0.004 & 0.002 & 0.044 \\ \text { parc2 } & \text { dorsal anterior cingulate cortex } & -0.003 & 0.001 & 0.040 \\ \text { parc6 } & \text { visual cortex } & 0.004 & 0.001 & 0.001 \\ \text { parc8 } & \text { parahippocampal cortex } & 0.003 & 0.001 & 0.036 \\ \text { parc9 } & \text { inferior frontal cortex } & -0.005 & 0.002 & 0.017 \\ \text { parc26 } & \text { dorsal medial prefrontal cortex } & -0.008 & 0.001 & <.001 \\ \text { parc30 } & \text { precuneus } & 0.004 & 0.001 & 0.017 \\ \text { parc33 } & \text { somatosensory cortex } & 0.006 & 0.001 & 0.001 \\ \text { parc37 } & \text { lingual gyrus } & -0.007 & 0.001 & <.001 \\ \text { parc41 } & \text { premotor cortex } & -0.004 & 0.001 & 0.019 \\ \text { parc49 } & \text { secondary somatosensory cortex } & -0.003 & 0.001 & 0.041 \\ \text { parc51 } & \text { anterior insula } & -0.005 & 0.001 & 0.017 \\ \text { parc52 } & \text { putamen } & 0.003 & 0.001 & 0.035 \\ \text { parc57 } & \text { auditory cortex } & 0.003 & 0.001 & 0.013 \\ \text { parc63 } & \text { superior temporal sulcus } & 0.003 & 0.001 & 0.017 \\ \text { parc67 } & \text { inferior frontal cortex } & 0.005 & 0.002 & 0.035 \\ \text { parc70 } & \text { occipitotemporal cortex } & -0.006 & 0.001 & 0.001 \\ \text { parc71 } & \text { insular cortex } & 0.003 & 0.001 & 0.035 \\ \text { parc76 } & \text { inferior occipital cortex } & -0.003 & 0.001 & 0.045 \\ \text { parc81 } & \text { somatosensory cortex } & 0.006 & 0.001 & <.001 \\ \text { parc86 } & \text { intraparietal sulcus } & -0.004 & 0.002 & 0.035 \\ \text { parc90 } & \text { premotor cortex } & 0.006 & 0.001 & 0.001 \\ \text { parc91 } & \text { intra parietal lobule } & -0.007 & 0.002 & 0.001\end{array}$

Supplementary Table 4 


\begin{tabular}{lllccc} 
Social Relationship Variable & Parcel Number & Brain Region & $b$ & $S E$ & $p$ \\
\hline Friendship & parc26 & dorsal medial prefrontal cortex & -0.022 & 0.006 & 0.026 \\
& parc33 & somatosensory cortex & 0.025 & 0.007 & 0.048
\end{tabular}

Knowing

$\begin{array}{lllll}\text { parc14 } & \text { supplementary motor cortex } & -0.007 & 0.002 & 0.043 \\ \text { parc26 } & \text { dorsal medial prefrontal cortex } & -0.006 & 0.002 & 0.011 \\ \text { parc33 } & \text { Somatosensory cortex } & 0.009 & 0.002 & 0.009 \\ \text { parc57 } & \text { auditory cortex } & 0.006 & 0.002 & 0.011 \\ \text { parc81 } & \text { somatosensory cortex } & 0.006 & 0.002 & 0.043 \\ \text { parc90 } & \text { premotor cortex } & 0.007 & 0.002 & 0.018 \\ \text { parc92 } & \text { thalamus } & 0.005 & 0.001 & 0.011\end{array}$

Perceived Similarity

$\begin{array}{lllll}\text { parc26 dorsal medial prefrontal cortex } & -0.009 & 0.002 & 0.001\end{array}$

Supplementary Table 5

Correlation Strength Between Social Relationship Variables

\begin{tabular}{ccccc} 
Social Relationship Variable & Friendship & Knowing & Liking & Similarity \\
\hline Friendship & 1 & 0.72 & 0.53 & 0.69 \\
Knowing & 0.72 & 1 & 0.37 & 0.69 \\
Liking & 0.53 & 0.37 & 1 & 0.55 \\
Similarity & 0.69 & 0.69 & 0.55 & 1
\end{tabular}



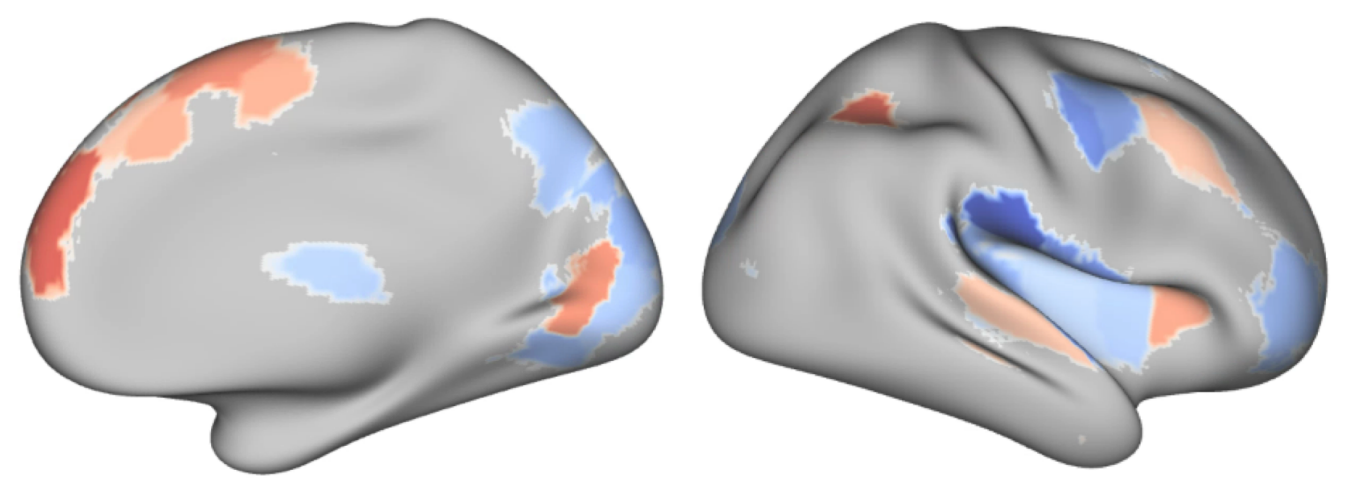

$-0.003$

Estimated Effect

Supplementary Figure 1. All four social relationship variables (friendship, knowing, liking and similarity) were aggregated into one metric of social relationship strength. Brain regions are shown here in which there was a significant relationship between the social relationship metric and similarity in brain response patterns when thinking of other group members. Warm colors indicate positive associations such that greater neural similarity to the group members was related to stronger relationship strength; cool colors indicated where there were negative associations. These results are highly consistent with the results from the original target congruence analysis where all four social relationship metrics were modeled separately. 

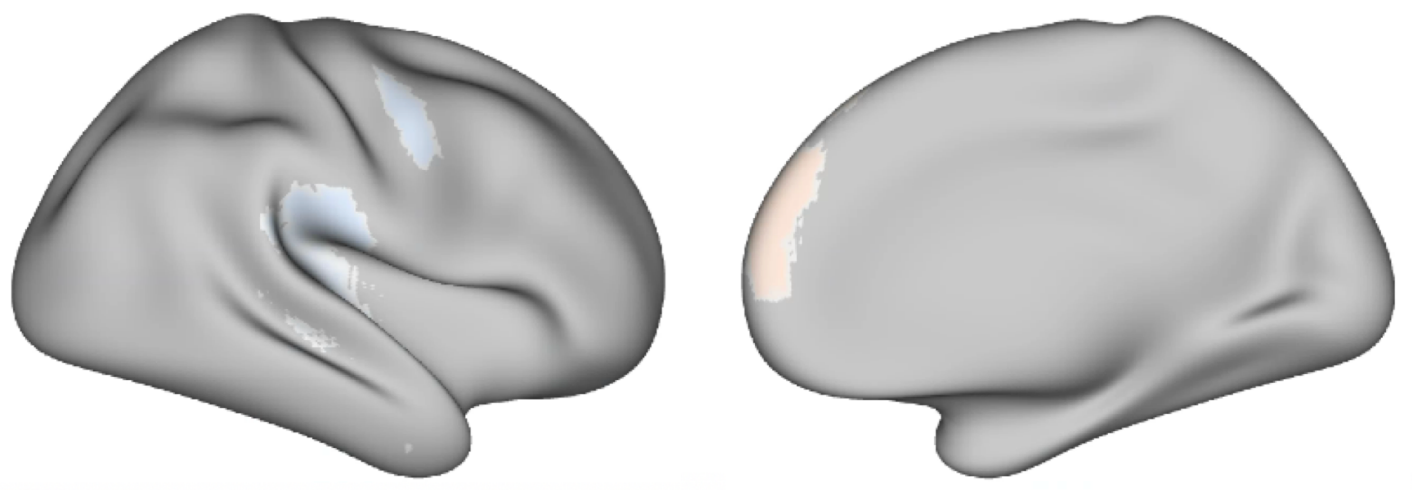

$-0.03$

Estimated Effect

0.03

Supplementary Figure 2. All four social relationship variables (friendship, knowing, liking and similarity) were aggregated into one metric of social relationship strength. Brain regions are shown here in which there was a significant relationship between the social relationship metric and brain similarity patterns to a group representation calculated by aggregating across all other members of the group. Warm colors indicate a positive relationship such that greater neural similarity to the group was related to stronger relationship strength, and cool colors where there was negative relationship. These results are highly consistent with the results from the original group aggregate analysis where all four social relationship metrics were modeled separately.
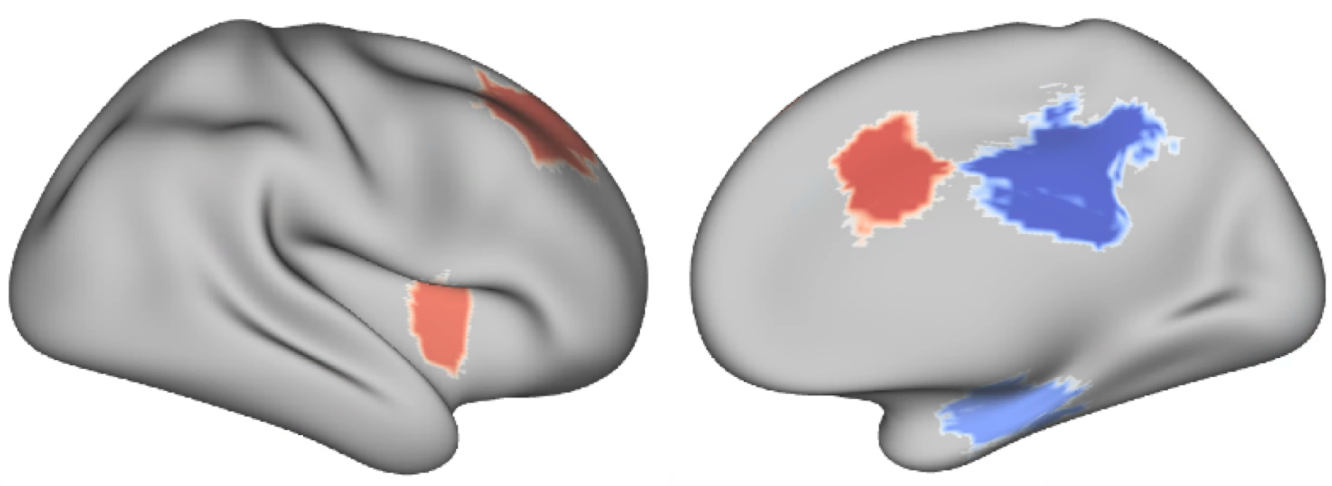
Supplementary Figure 3. Brain regions are shown here in which there was a significant relationship between the response congruence correlation strength and similarity in brain response patterns when thinking of other group members. Warm colors indicate a positive relationship such that greater neural similarity to the group members was related to stronger response congruence correlation strength, and cool colors where there was negative relationship. 